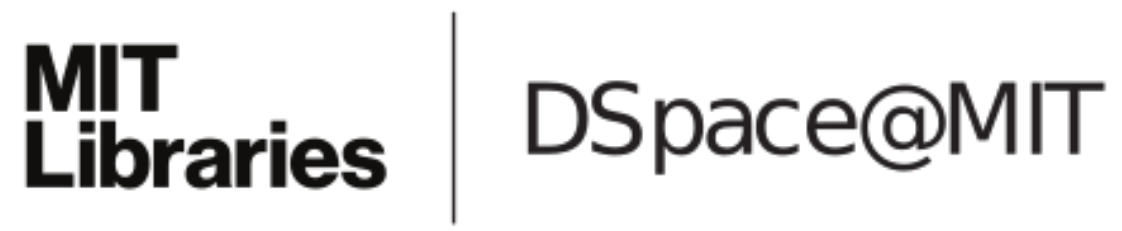

\author{
MIT Open Access Articles
}

Restoring the sense of touch

The MIT Faculty has made this article openly available. Please share how this access benefits you. Your story matters.

Citation: Anikeeva, Polina, and Ryan A. Koppes. "Restoring the Sense of Touch." Science 350, no. 6258 (October 15, 2015): 274-275.

As Published: http://dx.doi.org/10.1126/science.aad0910

Publisher: American Association for the Advancement of Science (AAAS)

Persistent URL: http://hdl.handle.net/1721.1/102695

Version: Author's final manuscript: final author's manuscript post peer review, without publisher's formatting or copy editing

Terms of Use: Article is made available in accordance with the publisher's policy and may be subject to US copyright law. Please refer to the publisher's site for terms of use. 


\section{Restoring the sense of touch}

Flexible circuitry mimics the way skin transduces pressure signals

By Polina Anikeeva ${ }^{1,2}$ and Ryan A. Koppes ${ }^{1,3}$

${ }^{1}$ Research Laboratory of Electronics, Massachusetts Institute of Technology, Cambridge, MA 02139 U.S.A. E-mail: anikeeva@mit.edu

${ }^{2}$ Department of Materials Science and Engineering, Massachusetts Institute of Technology, Cambridge, MA 02139 U.S.A.

${ }^{3}$ Department of Chemical Engineering, Northeastern University, Boston, MA 02115 U.S.A.

Amputation of damaged tissue is one of the oldest surgical techniques, reaching prevalence in the $16^{\text {th }}$ century (1). Improved emergency medicine has allowed more individuals to survive traumatic injuries as amputees, but prosthetic limbs remain the only means to restore any degree of function to these patients. Inadequate tactile feedback is a leading shortcoming of prosthetic limbs, but for artificial hands, just a few sensors that relay grasp pressure back to the user have an unprecedented impact on functionality, enabling delicate tasks (2). In addition to improved motor control, sensory stimulation could alleviate phantom limb pain, which affects $~ 80 \%$ of amputees (2). On page XXX of this issue Tee et al. (3) report a digital tactile system ("DiTacts") based on a lowpower flexible organic transistor circuit that transduces pressure stimuli into oscillating signals like those generated by skin mechanoreceptors.

A multilayered viscoelastic material, mammalian skin can stretch up to $\sim 125 \%$ from its resting dimensions and snap back into shape without any apparent loss in sensitivity to external stimuli such as pressure or temperature. Replicating skin mechanical and functional properties remains an elusive engineering challenge. Meanwhile, a rapidly expanding field of flexible electronics has made substantial strides, and complex circuits can now be produced on soft substrates. Advances in microcontact printing, inkjet deposition, and organic electronics have delivered stretchable and flexible, wearable, and even epidermal sensors (4-6).

These lightweight imperceptible devices, however, are often limited by the power requirements of directcurrent (DC) circuits. Biological mechanosensing circuits are characterized by low power consumption and minimal noise and drift afforded, in part, by frequency encoding of pressure information - higher pressures cause the mechanoreceptors in the skin to fire action potentials with higher frequencies. Inspired by this mechanism, Tee et al. develop a flexible organic ring-oscillator circuit that translates pressure input into a digital signal of 
variable frequency that scales with pressure. The DiTacts prototype uses a piezoresistive voltage divider element based on a microstructured elastomer layer impregnated with carbon nanotubes. Pressure on the elastomer reduces the distance and hence decreases the tunneling impedance between the nanotubes and the electrode. This, in turn, increases the voltage output of the divider, which translates into a lower gate delay of the inverters constituting the ring oscillator circuit thus increasing its oscillation frequency. Being inherently digital, DiTacts avoids noise and drift that plague DC-modulated circuits. The pressure-controlled frequency modulation eliminates the need for computing elements, as the output signal can be directly used to drive neural activity. Output frequencies up to $200 \mathrm{~Hz}$ were achieved at $11 \mathrm{~V}$ in a device with low-power consumption $(<20 \mu \mathrm{W})$, which will allow for future expansion of sensor density.

Prosthetic limbs remain largely limited by the technology connecting artificial driving and recording circuits to the biological sensory and motor pathways. In recent studies by Tyler, Micera, and co-workers, electrical stimulation of peripheral nerves improved the sensorimotor feedback in upper and lower limb prostheses $(2,7)$. A clinically relevant method, electrical stimulation, lacks the specificity needed to resolve efferent and afferent pathways (signals moving brain to muscle, and from skin to brain, respectively), let alone uniquely access specific fascicles (bundles of neuronal processes) within the peripheral nerves.

Optogenetics holds promise for improving signal transduction and cell specificity to the afferent nervous system. Through a multidimensional strategy of cell-specific promoters, opsins with distinct activation spectra, and spatiotemporally encoded optical stimulation, highly specific neural pathways can, in principle, be targeted. By optogentic decoupling of the sensory and motor pathways, future prostheses can be integrated with minimal invasiveness, avoiding the need for convoluted wiring and establishing direct feedback from an artificial limb to the brain.

Tee et al. outline a route to such optogenetic biointegration by combining the DiTacts platform with blue light-emitting devices and a custom-engineered channelrhodopsin 2 mutant bC1C2(s/v) with improved kinetics to better match the output frequencies of their system. In combination with the DiTacts platform, fast-spiking (parvalbumin-positive) mouse cortical neurons expressing this opsin, demonstrated a high correlation of their firing rate to increasing pressure, highlighting the close integration of machine and biology.

Although the advantages of optical neuromodulation over electrical stimulation are becoming more convincing, the future of genetic modification necessary for this technology to work in human patients remains unclear. Improved methods for induced pluripotency and on-demand stem cell differentiation (8) may someday enable optogenetic nerve-machine interfaces without the need for viral delivery of transgenes to patients (9). 
Furthermore, by triggering the innate mechanisms of neural repair (10) and plasticity, optical stimulation may allow for more intimate integration of synthetic tactile platforms with the patients' nervous systems.

Even with improved technology, broad adoption of advanced artificial limbs will be ultimately determined by the patient comfort. The latter will depend on the ability of the tactile feedback systems to faithfully represent the sense of touch, which remains a scientific challenge because of our incomplete understanding of proprioception. Biomimetic platforms, such as DiTacts artificial skin, are bound to aid the study of tactile perception, and with further development of dynamic response may enable prostheses that deliver a sense of touch.

\section{REFERENCES:}

1.M. Sachs, J. Bojunga, A. Encke, World J. Surg .23, 1088 (1999).

2.D. W. Tan et al., Sci. Transl. Med. 6, 257ra138 (2014).

3.B. C.-K. Tee et al., Science $\mathbf{x x x}$, xxx (2015).

4.M. Kaltenbrunner et al., Nature 499, 458 (2013).

5.Q. Li et al., Nature 523, 576 (2015).

6.D.-H. Kim et al., Science 333, 838 (2011).

7.S. Raspopovic et al., Sci. Transl. Med. 6, 222 ra19 (2014).

8.Z. P. Pang et al., Nature 476, 220 (2011).

9.J. B. Bryson et al., Science 344, 94 (2014).

10.S. Park et al., P. Anikeeva, Sci. Rep. 5, 9669 (2015).

Artificial skin for prosthetic devices. Tee et al. present the Digital Tactile System (DiTacts), a flexible, power-efficient mechanoreceptor array platform coupled to optogenetic neuromodulation for enabling the sense of touch in prosthetic limbs. 